Ther nodynami $\mathrm{c}$ properti es of an evaporat $i$ on process in sel $\mathrm{f}$-gravitating $\mathrm{N}$-body systens

\begin{tabular}{|c|c|}
\hline 著者 & $\begin{array}{l}\text { Konat su Nobuyoshi, Ki wat a Takahi ro, Ki mur a } \\
\text { Shi geo }\end{array}$ \\
\hline $\begin{array}{l}\text { j our nal or } \\
\text { publ i cat i on } \mathrm{title}\end{array}$ & $\begin{array}{l}\text { Physi cal Revi ew E - St at i st i cal, Nonl i near, } \\
\text { and Sof t Nat ter Physi cs }\end{array}$ \\
\hline vol une & 82 \\
\hline nunber & 2 \\
\hline page $r$ ange & 21118 \\
\hline year & $2010-08-17$ \\
\hline URL & ht t p: //hdl . handl e. net /2297/25206 \\
\hline
\end{tabular}




\title{
Thermodynamic properties of an evaporation process in self-gravitating $N$-body systems
}

\author{
Nobuyoshi Komatsu, ${ }^{1, *}$ Takahiro Kiwata, ${ }^{1}$ and Shigeo Kimura ${ }^{2}$ \\ ${ }^{1}$ Department of Mechanical Systems Engineering, Kanazawa University, Kakuma-machi, Kanazawa, Ishikawa 920-1192, Japan \\ ${ }^{2}$ The Institute of Nature and Environmental Technology, Kanazawa University, Kakuma-machi, Kanazawa, Ishikawa 920-1192, Japan
}

(Received 22 May 2010; published 17 August 2010)

\begin{abstract}
By means of $N$-body simulations, we consider self-gravitating open systems enclosed in a spherical container with semipermeable reflecting walls, in order to investigate the thermodynamics of the evaporation process in self-gravitating $N$-body systems (such as the escape of stars from globular clusters). To simulate the evaporation process, when the energy of a particle exceeds a certain threshold value, the particle passes through the semipermeable reflecting wall freely. We show that the thermodynamic properties of the evaporation process, such as the dependence of the temperature on energy, agree well with those of stellar polytropes, if the system is in an approximate virial equilibrium state. However, in a lower-energy region or for a rapid evaporation process, the thermodynamic properties deviate from those for the stellar polytrope. Nevertheless, we found that a negative specific heat occurs even in the lower-energy region or for a rapid evaporation process.
\end{abstract}

DOI: 10.1103/PhysRevE.82.021118

PACS number(s): 05.20.-y, 95.30.Tg, 05.70.-a, 45.50.Jf

\section{INTRODUCTION}

The statistical mechanics and thermodynamics of longrange attractive interacting systems exhibit several peculiar features such as negative specific heat and nonequilibrium nonextensive statistical mechanics and hence have been extensively studied [1-11]. It is known that the standard Boltzmann-Gibbs statistics are not valid in long-range attractive interacting systems, because of the existence of longrange potentials, and therefore the statistical mechanics and thermodynamics of self-gravitating systems have been investigated by many researchers [12-19] based on generalized statistics, e.g., Renyi's [20] and Tsallis' statistics [21] (see Ref. [22] and references therein). For example, Taruya and Sakagami have shown that an extremum state of Tsallis' entropy, i.e., a stellar polytrope, has a thermodynamic structure which implies a thermodynamic instability because of the negative specific heat [14-16].

In earlier studies, a system within an adiabatic wall, or the so-called Antonov problem [23], was typically investigated to examine the thermodynamics of self-gravitating $\mathrm{N}$-body systems such as globular clusters in galaxies. However, dynamical evolution causes stars to escape from globular clusters, because of the high energy of the stars or tidal forces of nearby galaxies [24-27]. That is, a mass and energy loss, or the so-called evaporation process, occurs in the dynamical evolution of the self-gravitating system. This evaporation process plays an important role in self-gravitating $N$-body systems. The process can drive a globular cluster toward a configuration with a high-density core [25], i.e., gravothermal catastrophe [23]. We expect that such a mass and energy loss affects not only the dynamical evolution of the system but also thermodynamic properties such as the incidence of negative specific heat.

However, from the viewpoint of thermodynamics, the influence of mass and energy loss has not been clarified in

\footnotetext{
*komatsu@t.kanazawa-u.ac.jp
}

self-gravitating $N$-body systems. (The energy loss has been discussed in a few studies, e.g., Posch and Thirring [11], and the present authors [28] have investigated the energy loss in long-range attractive interacting systems, using a circular or spherical container.) Moreover, it is not yet clear whether the stellar polytrope [14-16] can be applied to a nonequilibrium process with mass and energy loss, i.e., an evaporation process. In this context, we investigate the thermodynamic properties of the evaporation process in self-gravitating systems by $\mathrm{N}$-body simulations. (Of course, the thermodynamic properties discussed here are not in a thermodynamic equilibrium state, since self-gravitating systems are not in the exact equilibrium state. Accordingly, strictly speaking, a concept of specific heat should not be valid in the self-gravitating system. However, we apply such equilibrium concepts to a quasiequilibrium or nonequilibrium state and discuss the thermodynamic properties like in earlier studies.)

In this study, we consider a self-gravitating $N$-body open system enclosed in a spherical container with semipermeable reflecting walls. To mimic the evaporation process, when the energy of a particle exceeds a certain threshold value, the particle passes through the wall freely. We focus on the relationship between energy and temperature in the spherical wall, to examine the thermodynamic properties of the open system. We also discuss whether the stellar polytrope can be applied to a nonequilibrium process with mass and energy loss, i.e., the evaporation process.

The present paper is organized as follows. In Sec. II, we give a brief review of numerical techniques for simulating an evaporation process in self-gravitating $N$-body systems. In Sec. III, we present the simulation results and discuss the thermodynamic properties, e.g., the relationship between energy and temperature. In Sec. III A, we examine the influence of the initial energy on the evaporation process. In Sec. III B, we investigate the influence of an energy threshold to simulate the evaporation process, since the evaporation rate depends on the energy threshold. Finally, in Sec. IV, we present our conclusions. 


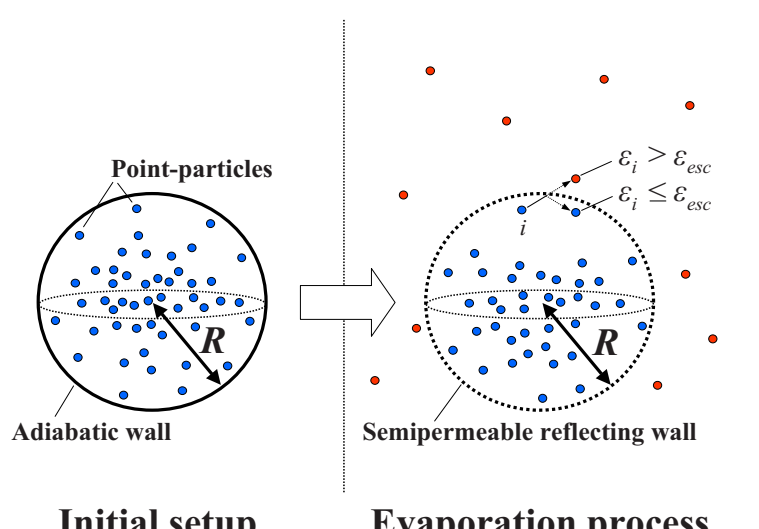

Initial setup

FIG. 1. (Color online) Setup for the $N$-body simulation. (Left) Initial setup; (right) evaporation process. To mimic an evaporation process, when the total energy $\varepsilon_{i}$ of the $i$ th particle exceeds an energy threshold $\varepsilon_{\text {esc }}$, the particle passes through the semipermeable reflecting wall freely.

\section{II. $N$-BODY SIMULATION TECHNIQUES}

In this section, we briefly review the $N$-body simulation method used in the present study $[28,29]$. We first consider a system consisting of $N$ point particles enclosed in a spherical container of radius $R$ with adiabatic walls as an initial setup (Fig. 1). To mimic an evaporation process, when the total energy of the $i$ th particle exceeds a certain threshold value, the particle passes through semipermeable reflecting walls. That is, we consider mass and energy loss in the container as a kind of evaporation process. (Hereafter we call this the evaporation process. The details are given later.) In our simulation, the whole self-gravitating system, i.e., the system inside and outside the sphere, evolves as time progresses. To simulate the whole system, we integrate a set of classical equations of motion for the particles interacting through the Plummer softened potential: $\Phi=-1 / \sqrt{r^{2}+r_{0}^{2}}$, where $r$ and $r_{0}$ represent the distance between particles and the softening parameter, respectively $[28,29]$. The total energy $E^{w}$ of the whole system is defined as

$$
E^{w}=E_{\mathrm{KE}}^{w}+E_{\mathrm{PE}}^{w}=\sum_{i}^{N} \frac{m_{i} v_{i}^{2}}{2}-\sum_{i<j}^{N} \frac{G m_{i} m_{j}}{\sqrt{r_{i j}^{2}+r_{0}^{2}}},
$$

where $E_{\mathrm{KE}}^{w}, E_{\mathrm{PE}}^{w}$, and $m_{i}$ represent kinetic energy, potential energy and the mass of the $i$ th point particle, respectively. $G$, $v_{i}$, and $r_{i j}$ represent the gravitational constant, the speed of the $i$ th particle and the distance between the $i$ th and $j$ th particles, respectively. The superscript notation $w$ represents a value of the whole system. The mass $m_{i}$ of each particle is set to be $m$.

In this paper, we focus on the thermodynamic properties of the system in the sphere, to examine the evaporation process. That is, we consider the system in the semipermeable reflecting wall as an open system. To define the total energy $E$ in the sphere, we apply Eq. (1) to $N_{s}$ particles in the sphere, where $N_{s}$ represents the number of particles in the sphere, $\left(r_{i}, r_{j}<R\right)$. In other words, we consider the influence of $N_{s}$ particles in the sphere, to compute the total energy $E$ in the sphere. The total rescaled energy $\varepsilon$ in the sphere is defined as

$$
\varepsilon=\varepsilon_{\mathrm{KE}}+\varepsilon_{\mathrm{PE}}=E \frac{R}{G M_{s}^{2}}=E \frac{R}{G\left(m N_{s}\right)^{2}},
$$

where $M_{s}$ represents the total mass in the sphere and $\varepsilon_{\mathrm{KE}}$ and $\varepsilon_{\mathrm{PE}}$ represent the rescaled kinetic and potential energies in the sphere, respectively. In this study, the unit of time is $\sqrt{R^{3} /(G m)}$. The units are set to be $G=R=m=1$, to ensure generality of the system. Therefore, the total rescaled energy $\varepsilon$ depends on the temporal energy $E$ and the temporal number $N_{s}$ of particles in the sphere. In our units, the temperature $\hat{T}$ of the system in the sphere is given by

$$
\hat{T}=\frac{2}{3 k_{\mathrm{B}}} \varepsilon_{\mathrm{KE}}=\frac{2}{3} \varepsilon_{\mathrm{KE}},
$$

assuming that the kinetic energy corresponds to the temperature and that Boltzmann's constant $k_{\mathrm{B}}$ is 1 [28]. We have confirmed that Eqs. (2) and (3) are suitable for examining the thermodynamic properties of an open system with a semipermeable reflecting wall.

In the present study, we consider a small system consisting of $N=250$ point particles inside and outside a spherical container of radius $R=1$. For simulating the $N$-body system, the set of equations of motion is integrated using Verlet's algorithm. To maintain the accuracy of our simulations, a time step of $\Delta t=10^{-5}$ is selected, based on a simulation with several different time steps $[28,29]$. In the present simulations, all interparticle forces are calculated directly at each time step $\Delta t$. All the results are averaged over 30 simulations with identically prepared initial setups, to observe the averaged behavior of the system [28].

For the evaporation process, we consider semipermeable reflecting walls to simulate mass and energy loss, as shown in Fig. 1. Such a semiconfined system is suitable for studying thermodynamic properties based on rescaled parameters, although this evaporation model is only one of the possible models. When the total energy $\varepsilon_{i}$ of the $i$ th particle in the sphere exceeds an energy threshold $\varepsilon_{\text {esc }}$, the particle freely passes through the semipermeable reflecting wall as if there was no wall. In contrast, when $\varepsilon_{i}$ is equal to or smaller than $\varepsilon_{\text {esc }}$, i.e., $\varepsilon_{i} \leqq \varepsilon_{\text {esc }}$, the spherical wall behaves like an adiabatic wall $[28,29]$. That is, the semipermeable reflecting wall behaves as follows:

$$
\text { behavior of wall }= \begin{cases}\text { no wall } & \left(\varepsilon_{i}>\varepsilon_{\mathrm{esc}}\right), \\ \text { adiabatic wall } & \left(\varepsilon_{i} \leq \varepsilon_{\mathrm{esc}}\right) .\end{cases}
$$

Note that the particles outside the sphere can pass through the wall freely. (The whole system, i.e., the system inside and outside the sphere, evolves as microcanonical ensemble simulations.) To calculate the rescaled energy $\varepsilon_{i}$, the total energy $E_{i}$ of the $i$ th particle in the sphere is defined as 


$$
E_{i}=\frac{m v_{i}^{2}}{2}-\sum_{j \neq i\left(r_{j}<R\right)}^{N_{s}} \frac{G m^{2}}{\sqrt{r_{i j}^{2}+r_{0}^{2}}} .
$$

The total energy $E_{i}$ of the $i$ th particle is rescaled using Eq. (2). Usually, the energy threshold $\varepsilon_{\text {esc }}$ should be 0 , since the particle can escape from the system when its kinetic energy is larger than the potential energy. The above operation for the present evaporation process slightly influences the total angular momentum of the system in the sphere. In fact, it is known that total angular momentum affects a phase transition of rotating systems, e.g., double clusters appear at high angular momenta in a spherical container [30]. Therefore, we have confirmed that the total angular momentum in the sphere is sufficiently smaller than that of the phase transition, during our evaporation process. That is, the phase transition induced by angular momentum should not occur in the present evaporation process.

In our simulations, the softening parameter $r_{0}$ for the Plummer softened potential is set to be $0.005 R$. Accordingly, the collapse and explosion energies for the system with an adiabatic wall are $\varepsilon_{\text {coll }} \approx-0.339$ and $\varepsilon_{\text {expl }} \approx 0.267$, respectively [31]. This means that if the total rescaled energy $\varepsilon$ of the uniform state becomes lower than $\varepsilon_{\text {coll }}$, the system should undergo a collapse to a core-halo state. In contrast, if the energy $\varepsilon$ becomes higher than $\varepsilon_{\text {expl }}$, the system should undergo an explosion.

In our units, the crossing time $\tau_{c}$ and the relaxation time $\tau_{r}$ for the present system are evaluated as $\tau_{c} \approx 1 / \sqrt{G \rho} \approx 0.1$ and $\tau_{r} \approx(0.1 N / \ln N) \tau_{c} \approx 0.6$, respectively, where $\rho$ represents the density of the system assuming a uniform density profile [28]. Accordingly, the collapse time of the present system is approximately 600, since the collapse time in a system with $N=125-250$ particles and $r_{0}=0.005 R$ is approximately $\sim 10^{3} \tau_{r}$ [32]. (The number $N_{s}$ of particles in the sphere decreases to approximately 50 in several simulations and, therefore, the collapse time of these systems should be slightly shorter than that for $N=250$. This is because the crossing and relaxation times for $N=50$ are evaluated as $\tau_{c}$ $\approx 0.3$ and $\tau_{r} \approx 0.4$, respectively.)

For the initial setup, we first prepare the self-gravitating system at an approximate virial equilibrium state, using adiabatic walls [28]. That is, all the particles are initially located in the sphere, as shown in Fig. 1. To obtain the approximate virial equilibrium state, the microcanonical ensemble simulation is continued over 10 units of time, i.e., for $t^{\prime}=10$, where $t^{\prime}$ represents the time for the initial setup. We have confirmed that the obtained system is in the approximate virial equilibrium state using the virial ratio $\alpha(t)$ of the system,

$$
\alpha(t)=\frac{2 E_{\mathrm{KE}}-4 \pi R^{3} P_{\mathrm{wall}}}{\left|E_{\mathrm{PE}}\right|},
$$

where $P_{\text {wall }}$ represents the pressure on the container wall by reflecting particles $[28,29]$. The virial ratio is 1 if the system is in the virial equilibrium state with pure gravitational potentials. A simulation of the evaporation process is carried out, based on the above initial setup. Note that the pressure on the reflecting wall at time $t$ can be evaluated as

$$
P_{\mathrm{wall}}(t)=\frac{\sum_{\hat{t}=t-t^{\prime \prime} / 2}^{\hat{t}=t+t^{\prime \prime} / 2} 2 m v_{\mathrm{r}}(\hat{t}) \sum_{\hat{=}=t-t^{\prime \prime} / 2}^{\hat{t}=t+t^{\prime \prime} / 2} m v_{\mathrm{r}}(\hat{t})}{4 \pi R^{2} t^{\prime \prime}}=\frac{\sum^{2} t^{\prime \prime}}{2 \pi}
$$

where $v_{\mathrm{r}}(\hat{t})$ is the summation of the radial components of the velocities of all particles reflected by the wall, at each time step $\hat{t}$ [31]. In general, fluctuations in the instantaneous pressure are larger than those in other macroscopic parameters. Therefore, in this study, the interval $t^{\prime \prime}$ in Eq. (7) is set to be $2000 \Delta t$ steps to reduce large fluctuations in the pressure.

We expect that the initial total energy $\varepsilon_{0}$ and energy threshold $\varepsilon_{\text {esc }}$ affect the evaporation process. Hence, we first examine the influence of the initial total energy. In Sec. III A, the initial energy $\varepsilon_{0}$ is varied between 0.2 and -0.2 . Therefore, the initial system should be in a stable or metastable state, since the initial energy is between $\varepsilon_{\text {coll }}$ and $\varepsilon_{\text {expl }}$ [31]. In the above simulation, the energy threshold $\varepsilon_{\text {esc }}$ is set to be 0 . In Sec. III B, to examine the influence of the energy threshold, $\varepsilon_{\text {esc }}$ is varied ranging from 0.0060 to -0.0050 . In this simulation, the initial energy $\varepsilon_{0}$ is set to be 0.1 .

In the present paper, the simulation time $t$ is usually 10 in our units, though in several cases, it is 12,20 , or 75 . Therefore, our simulation time should be shorter than the collapse time of the present system, $\sim 600$. In other words, in the evaporation simulation, we observe an early relaxation process before the collapse.

\section{RESULTS}

\section{A. Influence of the initial energy $\varepsilon_{0}$}

To investigate the influence of the initial energy $\varepsilon_{0}$, we first observe the time evolution of the number of particles in the sphere, for $\varepsilon_{0}=-0.2,-0.1,0.0,0.1$, and 0.2 . In this subsection, the energy threshold $\varepsilon_{\text {esc }}$ is set to be 0 , that is, when the kinetic energy of a particle is larger than the potential energy, the particle can escape from the sphere. (The particle outside the sphere can return into the sphere freely, in the present evaporation simulation.) The properties of the open system in the sphere are discussed in the following.

As shown in Fig. 2, the number $N_{s}$ of particles in the sphere decreases rapidly with time $t$ from the initial value of 250. This means that many particles escape from the spherical container. Thereafter, each curve gradually tends to a gentle decline. With increasing initial energy, $N_{s}$ decreases more rapidly because of high initial kinetic energy. To examine this closely, we plot the relationship between the initial evaporation rate and the averaged initial pressure on the wall. In Fig. 3, the initial evaporation rate $\left|d N_{s} / d t\right|$ is given by

$$
\left|\frac{d N_{s}}{d t}\right|=\left|\frac{N_{s}\left(\Delta t_{0}\right)-N_{s}(0)}{\Delta t_{0}}\right|,
$$

where $\Delta t_{0}$ is set to be $0.2 \tau_{c}(=0.02)$. To evaluate the averaged initial pressure on the wall, the pressure $P_{\text {wall }}$ on the wall is averaged over 30 simulations and also over $t^{\prime}=9-10$ in the simulation for the initial setup [28]. Moreover, the averaged initial pressure for each $\varepsilon_{0}$ is normalized by the pressure for $\varepsilon_{0}=0.2$. As shown in Fig. 3, the initial evaporation rate in- 


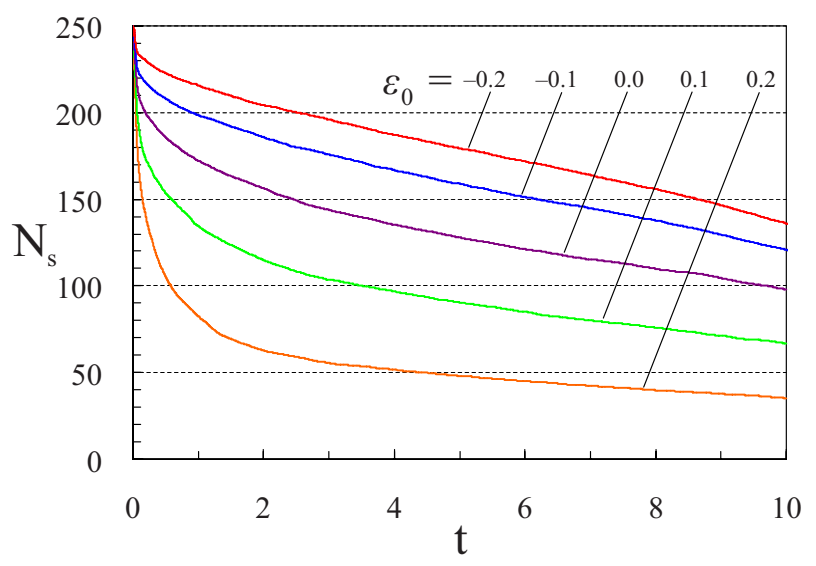

FIG. 2. (Color online) Time evolution of the number $N_{s}$ of particles in the sphere, for various initial energies $\varepsilon_{0}$. The energy threshold $\varepsilon_{\text {esc }}$ is 0 .

creases approximately linearly with the averaged initial pressure, in the stable or metastable state. That is, the lower the initial energy, the more moderate the evaporation process.

Using the above $N_{s}$ and Eq. (2), we examine typical time evolutions of the rescaled total and kinetic energies in the sphere. As shown in Fig. 4, the total energy $\varepsilon$ and the kinetic energy $\varepsilon_{\mathrm{KE}}$ decrease with time initially. However, the kinetic energy gradually increases and the total energy always decreases. This indicates that an incidence of negative specific heat occurs in the present evaporation process, since the kinetic energy corresponds to the temperature of the system at an approximate virial equilibrium state. The above result is consistent with the behavior appearing in a nonequilibrium process with nonadiabatic walls discussed in the previous study [28]. However, we emphasize that the number $N_{s}$ of particles in the sphere and the total mass in the sphere are not fixed in the present evaporation process, unlike in the previous study.

To examine the thermodynamics of the evaporation process, we plot the dependence of the temperature $\hat{T}$ on the

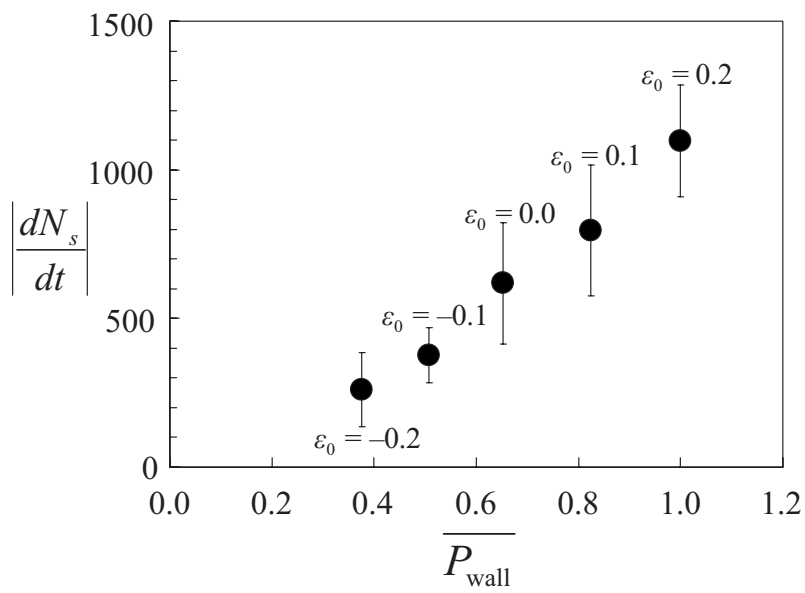

FIG. 3. Initial evaporation rate $\left|d N_{s} / d t\right|$ and averaged initial pressure on the wall. The horizontal axis represents the normalized averaged initial pressure on the wall. The error bars indicate the $68 \%$ confidence level in terms of the normal error distribution using 30 simulations.

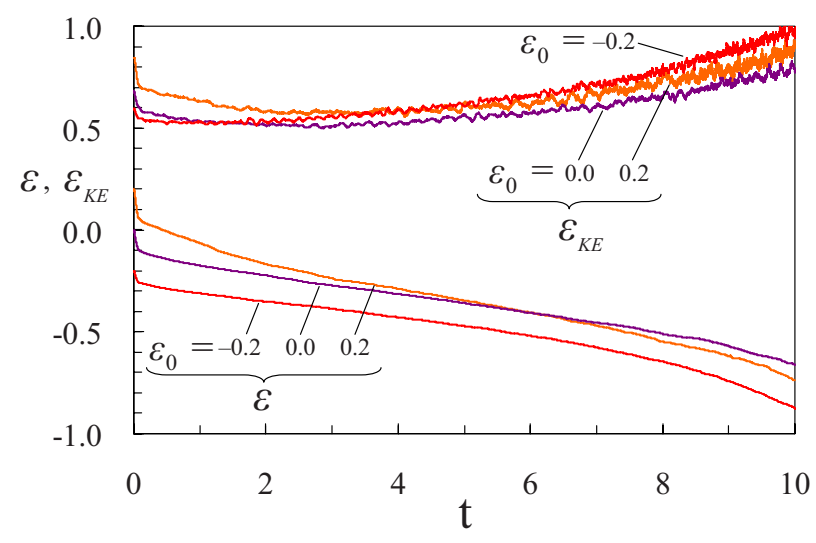

FIG. 4. (Color online) Time evolution of the total energy $\varepsilon$ and kinetic energy $\varepsilon_{\mathrm{KE}}$ in the sphere. The typical results are shown.

total energy $\varepsilon$, with various initial total energies $\varepsilon_{0}$. In Fig. 5, we display simulations starting from initial energies $\varepsilon_{0}$ of $-0.2,-0.1,0.0,0.1$, and 0.2 , represented by the open circles. Note that, in Figs. 5 and 6 , the simulation time for $\varepsilon_{0}=$ -0.1 and -0.2 is set to be 12 , to observe their properties appearing in lower-energy regions. The number of particles in the sphere for $\varepsilon_{0}=-0.2$ or -0.1 is approximately 100 , at the final low-energy state, i.e., at $t=12$.

As shown in Fig. 5, for $\varepsilon \gtrsim-0.2$, the total energy and temperature in the sphere decrease from the initial values. Accordingly, the system behaves like an ideal gas with a positive specific heat. In contrast, for $\varepsilon \lesssim-0.2$, the temperature increases with decreasing total energy. It is clearly demonstrated that a negative specific heat occurs in the present evaporation process, i.e., a nonequilibrium process with mass and energy loss. Interestingly, each simulation result is likely to lie on a common curve, except for $\varepsilon_{0}=0.2$.

As discussed in Ref. [28], a quasiequilibrium structure of stellar polytropes [14-17] is related to a nonequilibrium process appearing in a self-gravitating system with an energy

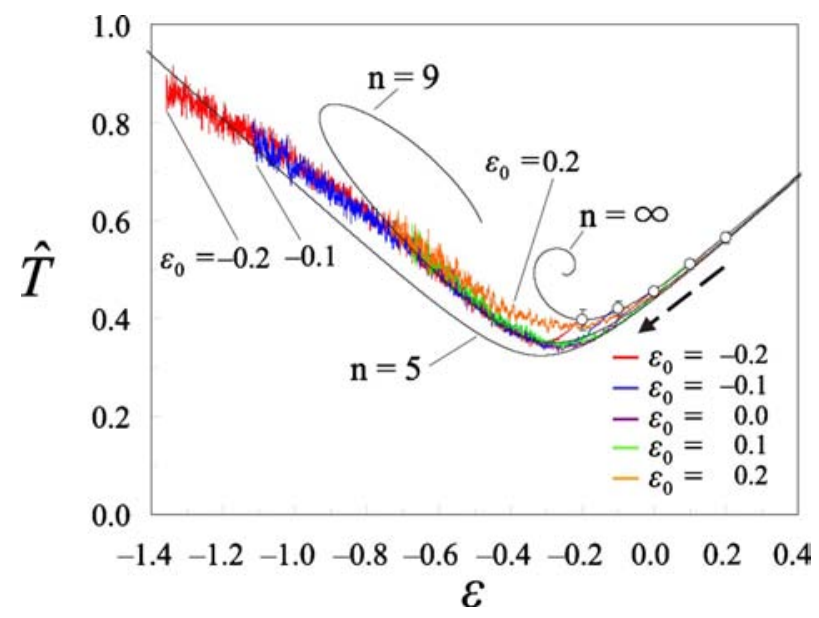

FIG. 5. (Color online) Dependence of the temperature $\hat{T}$ on the total energy $\varepsilon$ in the sphere, for various initial energies $\varepsilon_{0}$. The simulation starts from the initial total energy, represented by the open circle. The simulated system evolves as indicated by the arrow. Trajectories of Emden solutions with $n=5,9$, and $\infty$ are indicated for the stellar polytrope. 

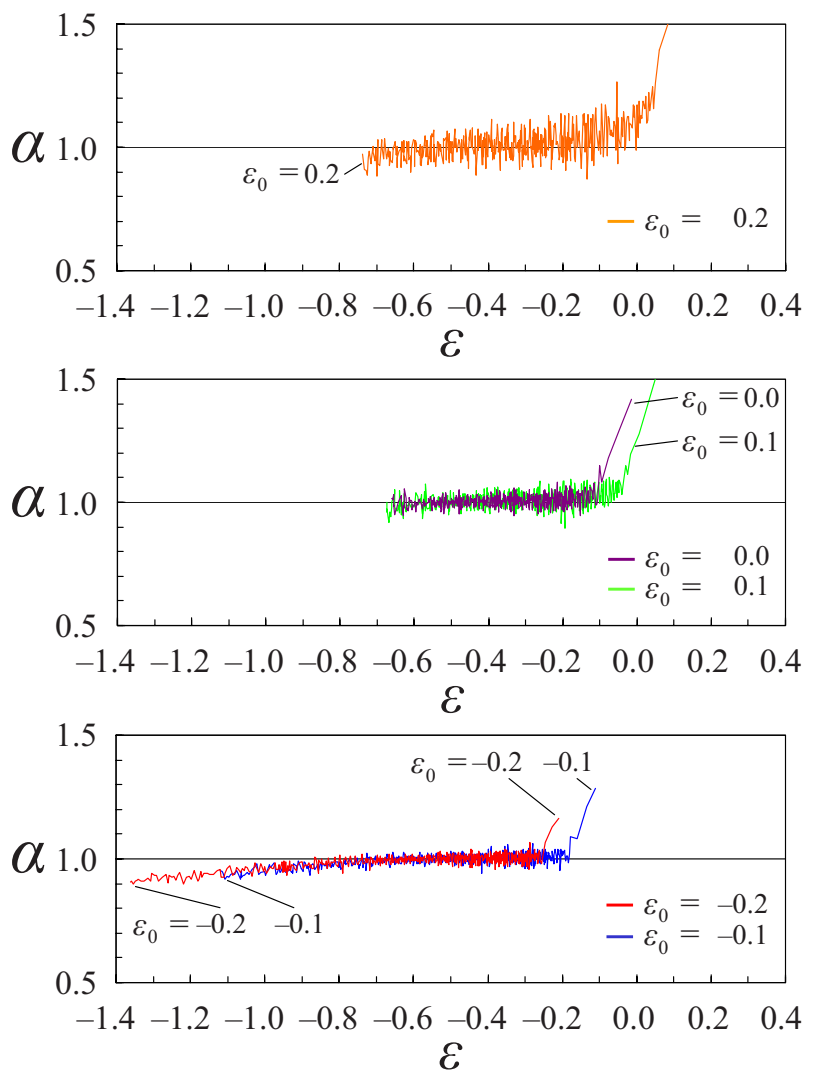

FIG. 6. (Color online) Dependence of the virial ratio $\alpha$ on the total energy $\varepsilon$ in the sphere, for various initial energies $\varepsilon_{0}$. (Top) $\varepsilon_{0}=0.2$, (middle) $\varepsilon_{0}=0.0$, and 0.1 , (bottom) $\varepsilon_{0}=-0.2$ and -0.1 . The simulated system evolves from right to left, as time progresses. The initial point represents the value at time $t=0.01$, to calculate the pressure $P_{\text {wall }}$ on the wall.

loss, under the restriction of constant mass. Therefore, we expect that the common curve appearing in the evaporation process is also related to the stellar polytrope, although the energy and mass vary during the evaporation process. In this context, we compare the simulation result with the stellar polytrope. To this end, we first briefly review the stellar polytrope [28]. (The stellar polytrope has been discussed extensively using various methods [33]. We employ the recent works of Taruya and Sakagami $[16,17]$ in those methods.) According to the works of Taruya and Sakagami [16,17], the polytropic relation can be given as

$$
P(r)=K_{n}\{\rho(r)\}^{1+1 / n},
$$

where $K_{n}, P(r)$, and $\rho(r)$ are the dimensional constant, isotropic pressure and density at radius $r$, respectively. The polytrope index $n$ is given by

$$
n=\frac{1}{1-q}+\frac{1}{2},
$$

where $q$ is the Tsallis entropic parameter. Note that, instead of standard linear means [12], normalized $q$ values [16] are selected as a statistical average [34]. We now consider a quasiequilibrium structure of the stellar polytropic system enclosed in a spherical container with adiabatic walls. Taruya and Sakagami have evaluated the total energy $E$ of a confined stellar system in terms of the total mass $M_{s}$, pressure $P_{e}$ and density $\rho_{e}$ at the boundary $r_{e}$. They also determined a plausible physical temperature $T_{\text {phys }}$, using the modified Clausius relation. Consequently, in our units, the total rescaled energy and temperature in the sphere can be given as

$$
\begin{gathered}
\varepsilon=\frac{E r_{e}}{G M_{s}^{2}}=\frac{1}{n-5}\left\{\frac{3}{2}\left(1-\frac{n+1}{v_{e}}\right)+(n-2) \frac{u_{e}}{v_{e}}\right\}, \\
\hat{T}=\frac{r_{e} T_{\text {phys }}}{G M_{s}^{2}}=\frac{n+1-2 u_{e}-v_{e}}{(n-5) v_{e}},
\end{gathered}
$$

where $u_{e}$ and $v_{e}$ are homology invariants at the wall, and $r_{e}$ corresponds to $R$ in the present study. The homology invariants are obtained from the Emden solutions [33]. In the above discussion, the total mass and energy are fixed.

Based on Eqs. (11) and (12), we plot trajectories of Emden solutions for polytrope indices of $n=5,9$ and $\infty$, as shown in Fig. 5. A polytrope index of $n=\infty$ corresponds to isothermal spheres or microcanonical ensembles. For $n>5$, the stellar polytrope within an adiabatic wall exhibits gravothermal instability. The trajectories for $n>5$ gradually change direction and finally spiral around a fixed point because of pure gravitational potentials [16]. It should be noted that the softening parameter for the Plummer softened potential is $r_{0}=0.005 R$ in our $N$-body simulations, while a pure gravitational potential is assumed for the stellar polytrope. Accordingly, we have to take into account the influence of the softening parameter.

As shown in Fig. 5 , for $\varepsilon \gtrsim-0.7$, the common $\varepsilon-\hat{T}$ curve, i.e., the $\varepsilon-\hat{T}$ curves for $\varepsilon_{0}=-0.2-0.1$, agrees well with the curve for a polytrope index of $n \sim 9$, except for a part of the spiral curve. In Ref. [28], a common curve appearing in the system with nonadiabatic walls agrees well with the curve for a polytrope index of $n \sim 5$ or rather $n>5$. Therefore, the present evaporation process is likely to be consistent with the stellar polytrope. Of course, the common curve moves upward slightly if the pure gravitational potential, i.e., $r_{0}=0$, is employed for the simulation [28]. Moreover, the stellar polytrope is assumed to be a quasiequilibrium structure under the restriction of constant energy and mass. However, we expect that the tendency toward a common curve is related to a kind of quasiattractor $[16,17]$. (The common curve appearing in the present study agrees with the stellar polytrope with $n$ $\sim 9$, unlike the previous study, for which it agrees with $n$ $\sim 5$. However, in the evaporation process, we found that the common curve is consistent with the stellar polytrope with $n>5$.)

In contrast, for $\varepsilon \lesssim-0.7$, the $\varepsilon-\hat{T}$ curves for $\varepsilon_{0}=-0.2$ and -0.1 gradually deviate from the curve for a polytrope index of $n \sim 9$. That is, the temperature of the $\varepsilon-\hat{T}$ curves gradually tends to be lower than that of the stellar polytrope with $n \sim 9$. We have confirmed that the present $\varepsilon-\hat{T}$ curves are consistent with an $\varepsilon-\hat{T}$ curve for $N=2500$, calculated from one ensemble simulation. To examine the cause of this de- 
viation, we observe the virial ratio $\alpha$ for various initial energies. Note that we do not discuss the local virial relation in the present study $[35,36]$.

As shown in Fig. 6, the system for $\varepsilon_{0}=0.2$ significantly deviates from 1 or the virial equilibrium state, probably because of rapid evaporation. This suggests that the $\varepsilon-\hat{T}$ curve for the rapid evaporation process is not on the common curve since the system is not in the virial equilibrium state. Except for $\varepsilon_{0}=0.2$ and the initial stages, the virial ratio is approximately 1 , when the energy is approximately larger than -0.7 , i.e., $\varepsilon \gtrsim-0.7$. However, the virial ratio gradually deviates from 1 when the energy is approximately smaller than -0.7 , i.e., $\varepsilon \lesssim-0.7$. That is, the system gradually tends to move away from the virial equilibrium state. In fact, the stellar polytrope assumes not only pure gravitational potentials but also quasiequilibrium states [16,17]. Accordingly, we conclude that the $\varepsilon-\hat{T}$ common curve deviates from the stellar polytrope if the system does not approach the virial equilibrium state. Alternatively, the kinetic energy may be unsuitable for describing the temperature of the system in such a situation.

Note that the quasiequilibrium state for the stellar polytrope with $n>5$ does not exist in lower-energy regions, because of the presence of pure gravitational potentials, i.e., $r_{0}=0$. For example, as shown in Fig. 5 , the $\varepsilon-\hat{T}$ curve has a spiral curve for $n=\infty$ or isothermal spheres. On the other hand, for an isothermal sphere with $r_{0}=0.005 R$, a mean-field phase diagram or an $\varepsilon-\hat{T}$ curve has high- and low-energy branches terminating at $\varepsilon_{\text {coll }} \approx-0.339$ and $\varepsilon_{\text {expl }} \approx 0.267$ [32]. That is, for $r_{0}=0.005 R$, the core-halo branch for the isothermal sphere exists in lower-energy regions. However, the evaporation process considered here is rapid and should be shorter than the collapse time for core-halo states. Therefore, the present system deviates from the quasiequilibrium state in the lower-energy region. We will discuss this later, using a density contrast.

\section{B. Influence of the energy threshold $\varepsilon_{\text {esc }}$}

In the previous subsection, the energy threshold $\varepsilon_{\text {esc }}$ was set to be 0 . In other words, a particle was able to escape from the open system when the kinetic energy of the particle was larger than the potential energy. However, it is expected that the energy threshold affects the present evaporation process. Therefore, in this subsection, we investigate the influence of the energy threshold $\varepsilon_{\text {esc }}$. To this end, the energy threshold $\varepsilon_{\text {esc }}$ is set to be $-0.0050,-0.0025,0,0.0025$, and 0.0060 . For $\varepsilon_{\text {esc }}=0.0025$ and 0.0060 , the simulation time is 20 and 75 , respectively, while the simulation time is 10 for the other energy thresholds. In this subsection, the initial energy $\varepsilon_{0}$ is set to be 0.1 . We have confirmed that our main result does not greatly depend on the initial energy, e.g., $\varepsilon_{0}=0.0$. Of course, the lower the initial energy, the more moderate the evaporation process, as discussed in Sec. III A. It should be noted that a particle outside the sphere can return into the sphere freely during our evaporation process.

We first observe the time evolution of the number of particles in the sphere, to examine the influence of the energy

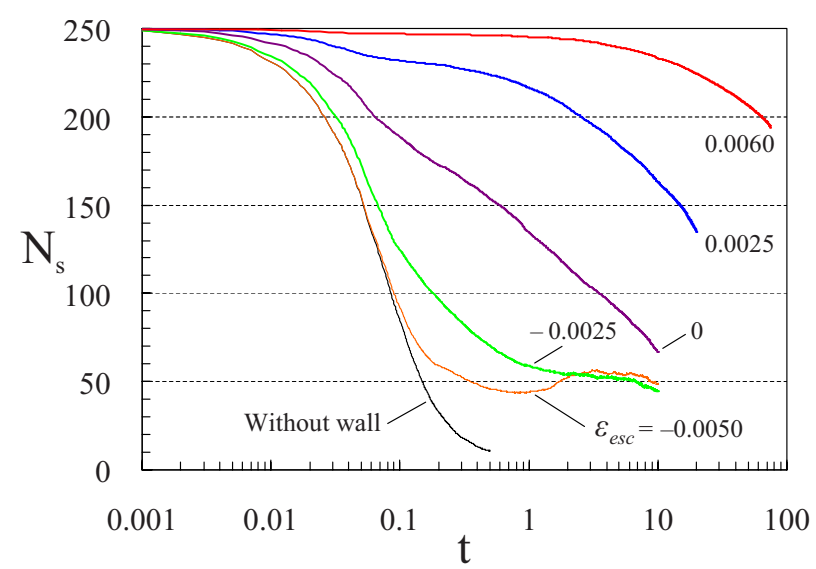

FIG. 7. (Color online) Time evolution of the number $N_{s}$ of particles in the sphere, for various energy thresholds $\varepsilon_{\text {esc }}$. The values with the curves indicate the energy threshold. The initial energy is $\varepsilon_{0}=0.1$.

threshold. (For reference, the result for the system without semipermeable walls is also plotted in Fig. 7. The initial condition for the system without walls is set to be the same as the other simulations, i.e., we first prepare the selfgravitating system at an approximate virial equilibrium state, using adiabatic walls. Note that, for the system without walls, we cannot discuss rescaled parameters, e.g., $\varepsilon$ or $\hat{T}$.)

As shown in Fig. 7, the number $N_{s}$ of particles in the sphere decreases with time. However, we found that $N_{s}$ for $\varepsilon_{\text {esc }}=-0.0050$ slightly increases after a rapid decrease. This indicates that several particles outside the sphere gradually return into the sphere, since the particles are within a gravitational sphere because of the negative energy threshold. As the energy threshold $\varepsilon_{\text {esc }}$ decreases, the number $N_{s}$ of particles in the sphere decreases more rapidly, just like the influence of the initial energy discussed in Sec. III A. That is, the lower the energy threshold, the more rapidly the particles can escape from the sphere. We can confirm this from the relationship between the energy threshold and the initial evaporation rate $\left|d N_{s} / d t\right|$, as shown in Fig. 8. In the present evaporation process, the initial evaporation rate for the sys-

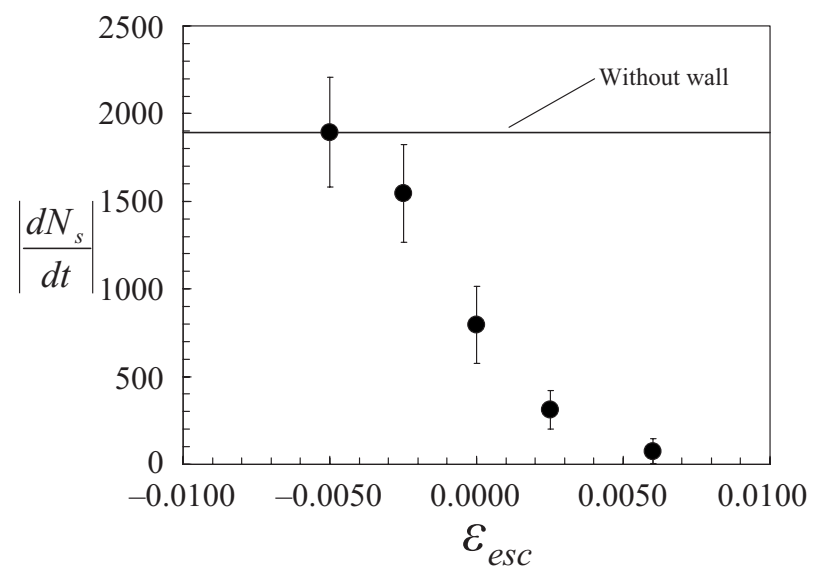

FIG. 8. Initial evaporation rate $\left|d N_{s} / d t\right|$ for various energy thresholds $\varepsilon_{\text {esc }}$. The horizontal line represents the initial evaporation rate for the system without walls. 


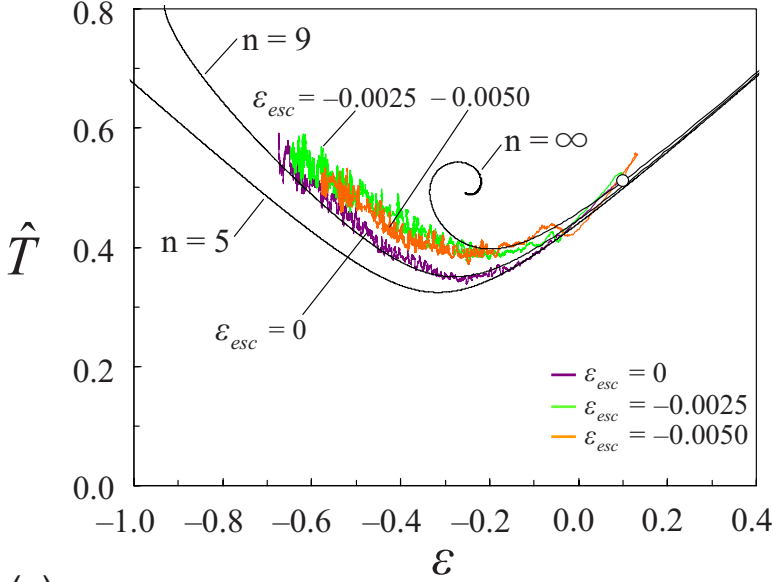

(a)

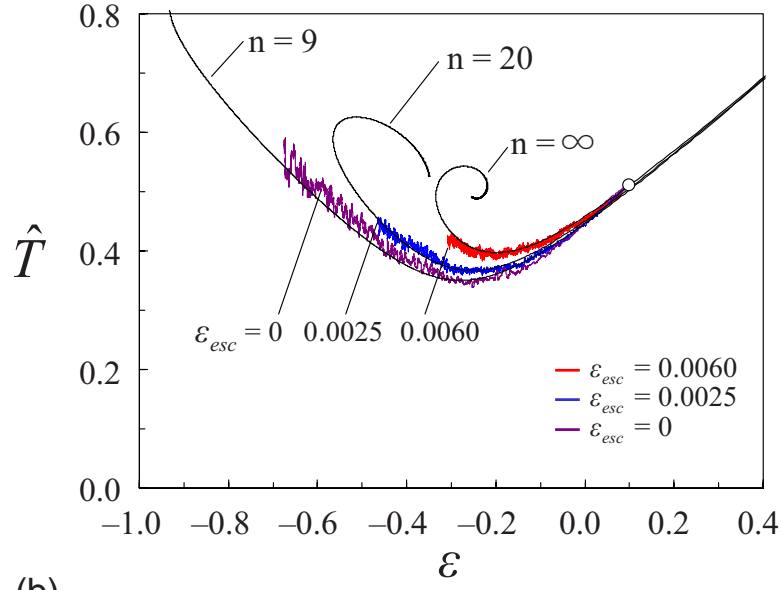

(b)

FIG. 9. (Color online) Dependence of the temperature $\hat{T}$ on the total energy $\varepsilon$ in the sphere, for various energy thresholds $\varepsilon_{\text {esc }}$. (a) Rapid evaporation $\left(\varepsilon_{\mathrm{esc}} \leq 0\right)$; (b) moderate evaporation $\left(\varepsilon_{\mathrm{esc}} \geq 0\right)$. The simulation starts from an initial energy $\varepsilon_{0}$ of 0.1 , which is represented by the open circle. Trajectories of the Emden solutions with $n=5,9,20$, and $\infty$ are indicated for the stellar polytrope. For $n=9$, a part of the spiral curve is not shown in this figure.

tem without walls approximately corresponds to the rate for $\varepsilon_{\text {esc }}=-0.0050$, which is smaller than $\varepsilon_{\text {esc }}=0$. This is probably because a spherical adiabatic wall is employed for our initial setup, i.e., the initial pressure on the wall is not 0 in the present study.

To examine the thermodynamic properties of the evaporation process, we plot the dependence of the temperature $\hat{T}$ on the total energy $\varepsilon$, with various energy thresholds $\varepsilon_{\text {esc }}$. In Figs. 9(a) and 9(b), each simulation starts from an initial energy of $\varepsilon_{0}=0.1$. As shown in Fig. 9(a), the $\varepsilon-\hat{T}$ curves for $\varepsilon_{\text {esc }}=-0.0025$ and -0.0050 deviate from the curve for $\varepsilon_{\text {esc }}$ $=0$. This is because the system does not approach the virial equilibrium state, due to rapid evaporation. However, we found that a negative specific heat, i.e., $d \varepsilon / d \hat{T}<0$, occurs even in such a rapid evaporation process. The above result is consistent with the $\varepsilon-\hat{T}$ curve for $\varepsilon_{0}=0.2$, which is shown in Fig. 5.

In contrast, as shown in Fig. 9(b), the result for $\varepsilon_{\text {esc }}$ $=0.0060$ agrees well with the $\varepsilon-\hat{T}$ curve for the isothermal sphere $(n=\infty)$, except for a part of the spiral curve. That is, the system behaves like the isothermal sphere, since the evaporation process is sufficiently moderate. With decreasing energy threshold, the curve gradually shifts toward the common curve, i.e., the stellar polytrope with $n \sim 9$. For example, the simulation results for $\varepsilon_{\mathrm{esc}}=0.0025$ and 0 agree well with the curves for the stellar polytropes with $n \sim 20$ and $\sim 9$, respectively. Therefore, we find that the thermodynamic properties of the moderate evaporation process are likely related to those for the stellar polytrope. (The influence of the energy threshold on the evaporation process is similar to that of cooling rates on a nonequilibrium process with nonadiabatic walls discussed in Ref. [28].)

If the system does not approach the virial equilibrium state in lower-energy regions, the $\varepsilon-\hat{T}$ curve deviates from the curve for the stellar polytrope, as discussed in Sec. III A. For instance, for $\varepsilon \lesssim-0.5$, the $\varepsilon-\hat{T}$ curve for $\varepsilon_{\mathrm{esc}}=0.0025$ deviates from the curve for the stellar polytrope with $n$ $\sim 20$ (not shown in the figure). This is probably because the quasiequilibrium state for uniform states does not exist in lower-energy regions. Moreover, the simulated system does not approach the core-halo states, since the present evaporation process is shorter than the collapse time for the corehalo state.

We now observe typical density profiles for $\varepsilon_{\text {esc }}=0$ and -0.0050 , to examine local properties in the evaporation process. From Fig. 9(a), we select three density profiles for which the temporal energy is approximately $\varepsilon=-0.1,-0.3$ and -0.5 . As shown in Figs. 10(a)-10(c), the densities for $\varepsilon_{\mathrm{esc}}=-0.0050$ are lower than those for $\varepsilon_{\mathrm{esc}}=0$. This is because the lower the energy threshold, the more rapidly the particles escape from the sphere. However, the density profiles for $\varepsilon_{\text {esc }}=0$ and -0.0050 are similar to each other. As the energy decreases [i.e., Figs. 10(a) to 10(c)], the density profile for $\varepsilon_{\text {esc }}=-0.0050$ gradually approaches the profile for $\varepsilon_{\mathrm{esc}}=0$, since the evaporation process tends to be moderate. However, the densities for $\varepsilon_{\mathrm{esc}}=-0.0050$ are always lower than those for $\varepsilon_{\text {esc }}=0$.

In Fig. 10, density profiles for the stellar polytrope are plotted as well. The line represents the Emden solution with $n=9$, which is fitted with the simulation result for $\varepsilon_{\mathrm{esc}}=0$, using Eq. (A.7) in Ref. [16]. Consequently, the density profile for the stellar polytrope can be well fitted with the simulation result for $\varepsilon_{\mathrm{esc}}=0$. This indicates that the density profile for the moderate evaporation process is consistent with the profile for the stellar polytrope.

As discussed above, the moderate evaporation process is likely related to the stellar polytrope. Therefore, for a closer comparison with the stellar polytrope, we observe the relationship between the density contrast $D=\rho_{c} / \rho_{e}$ and the negative of the total energy $\lambda(=-\varepsilon)[16,17]$. Here $\rho_{c}$ and $\rho_{e}$ represent the densities at the center and wall, respectively. To obtain the density contrast for the evaporation process, the density profile calculated from the Emden solution is fitted with the corresponding density profile of our simulations, as shown in Fig. 10. Based on the fitted curves, we evaluated the density contrast for the evaporation process. In Fig. 11, 

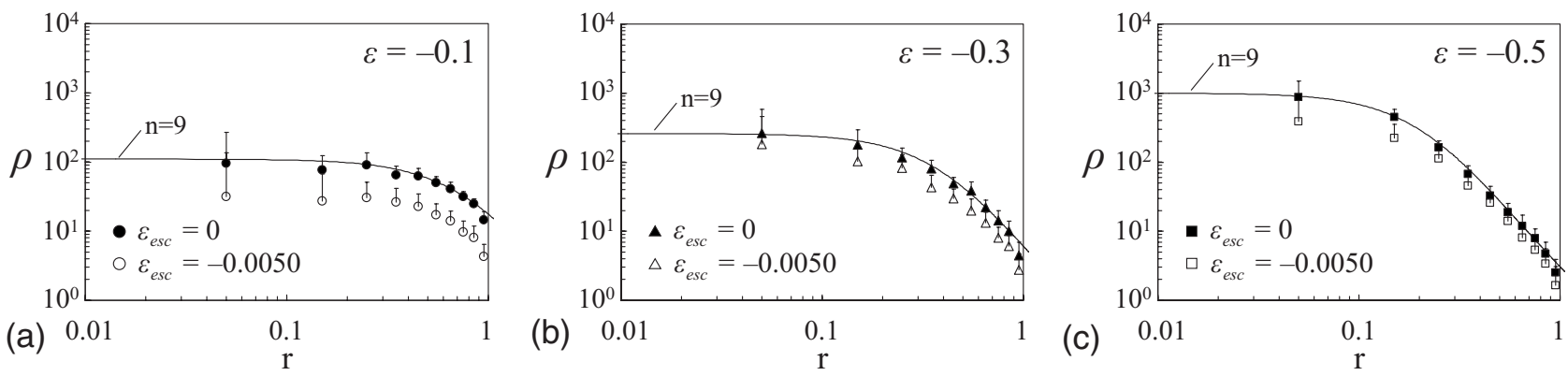

FIG. 10. Temporal density profiles of the evaporation process for temporal energies of approximately (a) $\varepsilon=-0.1$, (b) $\varepsilon=-0.3$, and (c) $\varepsilon=-0.5$. The closed and open symbols represent the simulation results for $\varepsilon_{\mathrm{esc}}=0$ and $\varepsilon_{\mathrm{esc}}=-0.0050$, respectively. The solid lines fitted with $\varepsilon_{\text {esc }}=0$ represent the stellar polytrope with $n=9$. For the evaporation process, the origin of $r$ is set to be the center of gravity.

typical density contrasts for the moderate evaporation process $\left(\varepsilon_{\text {esc }} \geq 0\right)$ are plotted as symbols. Note that the symbols in this figure represent evolutionary states. In other words, the states for the evaporation process evolve as indicated by the arrow.

We first focus on the density contrast for energy thresholds $\varepsilon_{\text {esc }}$ of $0(\square), 0.0025(\mathbf{\Delta})$ and $0.0060(\bigcirc)$, as shown in Fig. 11. The result for $\varepsilon_{\text {esc }}=0.0060$ agrees well with the curve for the stellar polytrope with $n=\infty$, since the evaporation process is sufficiently moderate. Similarly, the results for $\varepsilon_{\text {esc }}=0.0025$ and 0 agree well with the curves for $n \sim 20$ and $n \sim 9$, respectively. As the energy threshold decreases, the system for the evaporation process deviates from that for the isothermal sphere $(n=\infty)$ and approaches a certain system, which is likely related to the stellar polytrope with $n>5$.

Finally, we observe the density contrast for an energy threshold of $\varepsilon_{\text {esc }}=0$. That is, we examine the density contrast for initial energies of $\varepsilon_{0}=0.1(\square)$ and $-0.1(\diamond)$, as shown in Fig. 11 . For $\lambda \lesssim 0.6$ (i.e., $\varepsilon \gtrsim-0.6$ ), the results for $\varepsilon_{0}=0.1$ and -0.1 agree well with the $D-\lambda$ curves for $n \sim 9$. This result is consistent with the $\varepsilon-\hat{T}$ curve shown in Fig. 5 . Note that the

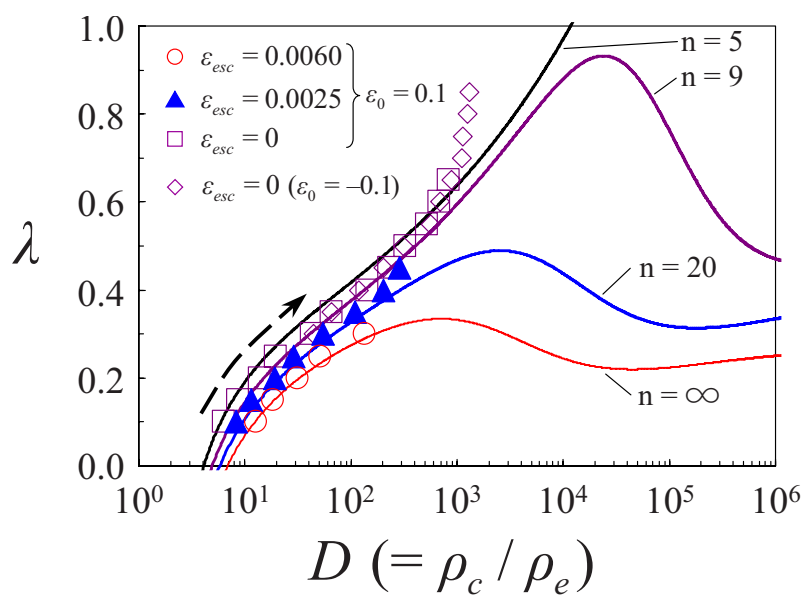

FIG. 11. (Color online) Density contrast $D\left(=\rho_{c} / \rho_{e}\right)$ and the negative of the energy $\lambda(=-\varepsilon)$. The symbols represent the simulation results for the evaporation process. The symbols show evolutionary states; that is, the states evolve as indicated by the arrow. The lines represent the stellar polytropes with $n=5,9,20$, and $\infty$. energy region discussed here should be slightly higher than that in Fig. $5(\varepsilon \gtrsim-0.7)$. In contrast, for $\lambda \gtrsim 0.6$ (i.e., $\varepsilon \lesssim$ $-0.6)$, the simulation results start deviating from the stellar polytrope with $n \sim 9$. This suggests that the evaporation process evolves rapidly, before the system has a sufficient highdensity core. In the present simulation, we have confirmed that the system deviates from the virial equilibrium state in such a lower-energy region, as discussed in Fig. 6.

These results indicate that the stellar polytrope can be applied to the present evaporation process, when the system is in the approximate virial equilibrium state. However, the stellar polytrope is not suitable for describing the evaporation process, if the evaporation process is too rapid or if the energy of the system is too low before collapse.

\section{CONCLUSIONS}

We considered a small $N$-body open system enclosed in a spherical container with semipermeable reflecting walls, to examine the thermodynamics of an evaporation process in self-gravitating systems. To mimic the evaporation process, when the energy of a particle exceeded a certain energy threshold, the particle could pass through the wall freely. Through the $N$-body simulation, we have clearly demonstrated that an incidence of negative specific heat occurs even in the present evaporation process or a nonequilibrium process with mass and energy loss. The dependence of the temperature on energy, i.e., the $\varepsilon-\hat{T}$ curve, is on a certain common curve in the evaporation process for various initial energies. The common curve is consistent with an $\varepsilon-\hat{T}$ curve for the stellar polytrope with $n>5$, when the system is in an approximate virial equilibrium state and not in a lowerenergy region.

Moreover, we examined the influence of the energy threshold on the evaporation process. We found that the thermodynamic properties agree well with those for the stellar polytrope, if the evaporation process is moderate. That is, the stellar polytrope is likely to be valid in the moderate evaporation process, although a fixed energy and mass are assumed for the stellar polytrope. However, in a rapid evaporation process or a lower-energy region, the simulated evaporation process deviates from the stellar polytrope, since the system 
should not approach the virial equilibrium state sufficiently. Nevertheless, the negative specific heat occurs in the evaporation process under such conditions. In other words, we have shown that peculiar thermodynamic properties appear in strong nonequilibrium phenomena through the evaporation simulation. The present study opens up a new theoretical approach for examining strong nonequilibrium phenomena appearing in self-gravitating systems.
[1] J. L. Lebowitz and E. H. Lieb, Phys. Rev. Lett. 22, 631 (1969).

[2] W. Thirring, Z. Phys. 235, 339 (1970).

[3] M. K. H. Kiessling, J. Stat. Phys. 55, 203 (1989).

[4] T. Padmanabhan, Phys. Rep. 188, 285 (1990).

[5] H. A. Posch, H. Narnhofer, and W. Thirring, Phys. Rev. A 42, 1880 (1990).

[6] D. Lynden-Bell, Physica A 263, 293 (1999).

[7] V. P. Youngkins and B. N. Miller, Phys. Rev. E 62, 4583 (2000).

[8] E. Follana and V. Laliena, Phys. Rev. E 61, 6270 (2000).

[9] I. Ispolatov and E. G. D. Cohen, Phys. Rev. Lett. 87, 210601 (2001).

[10] W. Thirring, H. Narnhofer, and H. A. Posch, Phys. Rev. Lett. 91, 130601 (2003).

[11] H. A. Posch and W. Thirring, Phys. Rev. Lett. 95, 251101 (2005).

[12] A. Plastino and A. R. Plastino, Phys. Lett. A 174, 384 (1993).

[13] R. Silva and J. S. Alcaniz, Phys. Lett. A 313, 393 (2003).

[14] A. Taruya and M. Sakagami, Phys. Rev. Lett. 90, 181101 (2003); Mon. Not. R. Astron. Soc. 364, 990 (2005).

[15] A. Taruya and M. Sakagami, Physica A 307, 185 (2002); 318, 387 (2003).

[16] A. Taruya and M. Sakagami, Physica A 322, 285 (2003).

[17] M. Sakagami and A. Taruya, Continuum Mech. Thermodyn. 16, 279 (2004).

[18] A. Nakamichi and M. Morikawa, Physica A 341, 215 (2004).

[19] P. H. Chavanis, Astron. Astrophys. 432, 117 (2005).

[20] A. Renyi, Probability Theory (North-Holland, Amsterdam, 1970).

[21] C. Tsallis, J. Stat. Phys. 52, 479 (1988).

[22] C. Tsallis, Introduction to Nonextensive Statistical Mechanics: Approaching a Complex World (Springer, New York, 2009).
[23] V. A. Antonov, in Dynamics of Globular Clusters, IAU Symposium No. 113, edited by J. Goodman and P. Hut (Reidel, Dordrecht, 1985); Vestn. Leningrad Univ. 7, 135 (1962).

[24] J. Binney and S. Tremaine, Galactic Dynamics (Princeton University Press, Princeton, 1987).

[25] D. Fanelli, M. Merafina, and S. Ruffo, Phys. Rev. E 63, 066614 (2001)

[26] D. Fanelli and S. Ruffo, Physica A 305, 253 (2002).

[27] M. Lemou and P. H. Chavanis, Physica A 389, 1021 (2010).

[28] N. Komatsu, S. Kimura, and T. Kiwata, Phys. Rev. E 80, 041107 (2009); J. Phys.: Conf. Ser. 201, 012009 (2010).

[29] N. Komatsu, T. Kiwata, and S. Kimura, Physica A 387, 2267 (2008); 388, 1344 (2009); 388, 639 (2009).

[30] E. V. Votyakov, H. I. Hidmi, A. De Martino, and D. H. E. Gross, Phys. Rev. Lett. 89, 031101 (2002).

[31] I. Ispolatov and M. Karttunen, Phys. Rev. E 68, 036117 (2003)

[32] I. Ispolatov and M. Karttunen, Phys. Rev. E 70, 026102 (2004).

[33] S. Chandrasekhar, Introduction to the Study of Stellar Structure (Dover, New York, 1939).

[34] Equation (10) is different from the standard relationship between the polytrope index $n$ and the $q$ parameter, i.e., $n=\frac{3}{2}$ $+\frac{1}{q-1}$ [12]. This is because the relationship between $n$ and $q$ depends on a choice of the statistical average [14]: standard linear means [12] or normalized $q$ values [16]. In this study, the normalized $q$ value [16] is selected as the statistical average.

[35] O. Iguchi, Y. Sota, A. Nakamichi, and M. Morikawa, Phys. Rev. E 73, 046112 (2006).

[36] Y. Sota, O. Iguchi, T. Tashiro, and M. Morikawa, Phys. Rev. E 77, 051117 (2008). 